\title{
tRNA processing, modification, and subcellular dynamics: past, present, and future
}

\author{
ERIC M. PHIZICKY ${ }^{1}$ and ANITA K. HOPPER ${ }^{2}$ \\ ${ }^{1}$ Department of Biochemistry and Biophysics, Center for RNA Biology, University of Rochester School of Medicine, Rochester, New York 14642, USA \\ ${ }^{2}$ Department of Molecular Genetics, Center for RNA Biology, The Ohio State University, Columbus, Ohio 43210, USA
}

tRNA research was such a small field in 1995 that we used to quip that we could have a meeting of the entire American community interested in tRNA processing and modification in one of our living rooms. Research since the 1960's had yielded the crucial role of tRNAs in translation, and contributed heavily to our understanding of RNA structure and chemistry. We knew the structure of three tRNA species, for many years the only structures of functional RNAs, and we knew the identity, position, and physical properties of many of the myriad of tRNA modifications in several experimental organisms. Moreover, tRNA research from the late 1970's and early 1980's had ushered in the era of RNA splicing and RNA catalysis, had unraveled large parts of the puzzle of the second genetic code controlling how tRNAs are specifically charged by their cognate synthetases, and had uncovered some of the important mechanisms of charging fidelity. To most observers, tRNA was mostly a solved problem.

The past 20 years have witnessed a resurgence of interest in tRNA research. In the old view, circa 1995, tRNA was made once, during which time it exited the nucleus to participate in translation in the cytoplasm, virtually endlessly due to its hyperstable nature, with modifications that were suggested to fine-tune tRNA function. No more. The new view of tRNA is of an RNA class that is amazingly dynamic, is subject to significant quality controls during and after synthesis, is a significant mediator of regulation, and is implicated in several human diseases. We describe these and other developments below, and then speculate on the future of tRNA research.

tRNA processing, modification, and intracellular transport are far more complex and interesting than our view in 1995. At that time, eight genes involved in tRNA biogenesis after transcription had been identified in the yeast Saccharomyces cerevisiae: TRM1, TRL1, MOD5, SEN2, RPR1, POP1, LOS1, and TRM2; as well as RNA1, which would turn out to be only indirectly involved in tRNA processing, although it was instrumental in the study of tRNA trafficking and

Corresponding author: eric_phizicky@urmc.rochester.edu

Article and publication date are at http://www.rnajournal.org/cgi/doi/ 10.1261/rna.049932.115. Freely available online through the RNA Open Access option.
tRNA splicing. Today, this list includes 100 genes in S. cerevisiae, including 74 genes involved in modification of the 25 chemically distinct modifications that are found in 36 positions in yeast cytoplasmic tRNAs. In E. coli and other organisms there has been a similar explosion in our knowledge of the machinery for tRNA biogenesis.

The past 20 years have also witnessed new appreciation of the crucial roles of many modifications in tRNA function, and their linkage to human disease. In S. cerevisiae, mutations in genes affecting 16 of the 25 modifications result in a distinct phenotype, including lethality for three mutants, poor growth for four mutants, and temperature sensitivity for two other mutants. In $S$. pombe, similar distinct phenotypes have been observed, and in metazoan model systems lack of specific modifications are associated with learning defects, male infertility, developmental defects, altered aging, protection against stress induced tRNA cleavage, and alteration of Dicer-2 dependent siRNA pathway function. In humans, lack of a number of different modifications has been associated with neurodegenerative diseases including autosomal recessive intellectual disability, X-linked intellectual disability, and possibly familial dysautonomia, as well as with the mitochondrial diseases MERFF and MELAS.

Moreover, the specific translation functions of many modifications have begun to emerge. Structural biology and biochemical analysis has provided a detailed look at the role of specific modifications in decoding in the ribosome A-site, in aspects of translation initiation, and in charging of specific tRNAs, and genetics has shown the pivotal role of several anticodon loop modifications in reading frame maintenance and decoding efficiency.

Substantial links have also been made between the tRNA processing and modification pathways and other aspects of cell physiology. For example, the tRNA splicing machinery is directly linked to the unfolded protein response in yeast and vertebrates due to shared components. In addition, there are crucial interactions between enzymes of the modification machinery and other cellular machinery, including the sterol

(C) 2015 Phizicky and Hopper This article, published in RNA, is available under a Creative Commons License (Attribution-NonCommercial 4.0 International), as described at http://creativecommons.org/licenses/by-nc/4.0/. 
biosynthesis and endosome recycling pathways in yeast, and the DNA repair machinery in yeast and humans.

Recent discoveries have also provided several cases of unexpected processing pathway diversity. For example, two distinct pathways have been defined for the ligation step of tRNA splicing following endonucleolytic excision of the intron to generate half-molecules. Yeast, plants, and some animals use a complicated $5^{\prime}$-phosphate ligase, which opens the $2^{\prime}-3^{\prime}$ cyclic phosphate end to form a $2^{\prime}$ - phosphate, phosphorylates and activates the $5^{\prime}$ end, and joins the halves to generate mature tRNA with a splice junction $2^{\prime}$-phosphate, which is then transferred to NAD by a phosphotransferase. By contrast, in archaea, humans, and a number of different metazoans, the ligation step involves a single enzyme that joins the half-molecules by activating the $3^{\prime}$ phosphoryl end, followed by ligation.

A second example of pathway diversity is that of RNase $\mathrm{P}$ in removal of the $5^{\prime}$ leader from tRNAs. In bacteria, archaea, and a large number of eukaryotes, $5^{\prime}$ leader removal is catalyzed by the RNA component of RNase P, aided by one or more protein co-factors; however, it is now known that this reaction is catalyzed only by protein in the nucleus and organelles of some plants, in the nucleus of some protists, and in mitochondria of some animals. Yet a third example of pathway diversity is the differing mechanisms for $2^{\prime}-O$ methylation and pseudouridylation, employing single proteins or small protein complexes in some cases, and RNAguided ribonucleoprotein complexes in other cases.

Remarkably, divergent processing pathways can often be substituted for one another. Thus, in yeast the human $3^{\prime}$ phosphate splicing ligase enzyme can substitute for the $5^{\prime}$ phosphate ligase (and the phophotransferase) to catalyze the ligation step of both tRNA splicing and the unusual HAC1 mRNA splicing reaction that is central to the unfolded protein response; and the single RNase $\mathrm{P}$ protein enzyme from Arabidopsis can substitute for the entire 10 subunit yeast RNase P complex, with no untoward growth defects.

This past generation of tRNA research has also revealed the critical importance of tRNA trafficking. The traditional view of the nuclear pore as a one-way valve for tRNA biogenesis has been upended. Amazingly, during steady state growth in yeast, intron-containing tRNAs wend their way from their site of synthesis in the nucleolus to the inner nuclear membrane for a modification, and across the nuclear pore to the outer mitochondrial surface for splicing, where the endonuclease resides in yeast, but not in vertebrates. Once spliced in the cytoplasm, the tRNA returns to the nucleus by a trafficking pathway called retrograde nuclear import where additional modifications are sometimes added, and is reexported to the cytoplasm for final modifications and translation. In addition, mitochondrial import of tRNAs is now known to be important or essential for mitochondrial function in a large number of eukaryotes.

Recent research efforts have also uncovered multiple layers of tRNA quality control in yeast and likely other organisms.
The yeast nuclear surveillance pathway acts to degrade pretRNA $_{i}^{\text {Met }}$ lacking $\mathrm{m}^{1} \mathrm{~A}$ in the T-loop or bearing defects in $3^{\prime}$ end processing, by polyadenylation of the pre-tRNA by the TRAMP complex, followed by $3^{\prime}-5^{\prime}$ exonucleolytic degradation by the nuclear exosome. The nuclear export machinery preferentially acts to export pre-tRNAs with $5^{\prime}$ and $3^{\prime}$ processed ends. The rapid tRNA decay (RTD) pathway degrades specific mature tRNA species lacking one or more of several critical modifications, or with a destabilized acceptor and/or T-stem, by $5^{\prime}-3^{\prime}$ exonucleolytic degradation of tRNAs by Xrn1 and/or Rat1. Finally, the retrograde nuclear import tRNA pathway targets tRNAs that are hypomodified or that have extended $5^{\prime}$ or $3^{\prime}$ ends for subsequent processing or degradation in the nucleus. Although these pathways have not been investigated in detail in metazoan cells, recent evidence suggests that the RTD pathway acts in human cells on initiator tRNAs subject to heat shock, and that mouse cells lacking certain modifications have reduced levels of tRNA species.

It has also become increasingly clear in the last 20 years that tRNA is a major factor during various stress responses. It had been known for some time that amino acid starvation in yeast and humans leads to uncharged tRNA that triggers the general amino acid control response by derepressing GCN4 translation to reprogram cellular transcription. In the last 20 years several additional intricate stress responses have been uncovered. In yeast, DNA damaging treatments, or starvation for amino acids, phosphate, or glucose triggers accumulation of cytoplasmic tRNAs in the nucleus, and likewise, in humans amino acid starvation leads to nuclear accumulation of previously cytoplasmic tRNAs. The levels of several tRNA modifications are known to vary in response to a number of different cellular stresses in yeast and other organisms, and in yeast altered modification levels are known to lead to altered translation and cellular physiology. In addition, tRNA synthesis is now known to be regulated in response to nutrient and other signaling pathways.

One distinct surprise of the last decade was the observation that an important part of the stress response involved tRNA fragments. Stress treatments in different organisms elicit tRNA cleavage by nucleases such as angiogenin to produce tRNA-derived stress fragments, which inhibit translation in mammalian cells and archaea, protect against apoptosis in mammalian cells, and inhibit the siRNA pathway in Drosophila. Furthermore, oxidative stress results in cleavage of the CCA end in mammalian cells, leading to a transient inhibition of tRNA function in translation. In addition, a specific tRNA-derived fragment is associated with cancer cell lines and appears to promote cell proliferation, as does overproduction of $\mathrm{TRNA}_{\mathrm{i}}^{\mathrm{Met}}$.

With all this evidence for crucial roles of tRNAs, tRNA fragments, and tRNA processing machinery in diverse biological systems, what does the next 20 years of tRNA research hold in store?

Based on the pace of recent results, it seems likely that four developments will occur. First, the cause of the many human 
diseases associated with defects in modifications will be elucidated. The specific tRNA(s) that are affected will be identified, the effects of these hypomodified tRNAs on translation will be enumerated, and the reasons why the diseases are associated with the nervous system will be understood. Similarly, we will understand why mutations in the tRNA splicing endonuclease lead to the neurodegenerative disease pontocerebellar hypoplasia, and we will understand the cause of the many mitochondrial diseases associated with tRNA gene mutations, as the roles of modifications and the determinants of tRNA function become better defined. Second, study of the regulation of tRNA processing and modification during growth, development, differentiation, and stress is likely to uncover new mechanisms of quality control and of the involvement of tRNA during regulation, much like the discovery of tRNA fragments and their regulatory effects. Third, there will be many more cases in which specific tRNA isodecoders (tRNA variants with the same anticodon) or specific modifications are implicated in biology, like the recent discovery of a mouse tRNA ${ }^{\text {Arg }}$ isodecoder lacking in certain mouse strains that is implicated in neurodegeneration in mutant backgrounds. Fourth, there will be mechanistic understanding of the roles of individual tRNA species and tRNA residues at every kinetic step of the translation cycle and each location within the ribosome, and mechanistic understanding of why and how specific tRNAs and tRNA combinations alter individual steps of translation.
To accomplish many of these future discoveries, there will be much improved methodology for evaluating the contribution of every individual tRNA isodecoder to tRNA expression and regulation, and new methods will be developed to monitor the function and occurrence of modifications in all RNAs, like the recent development of methods for analysis of inosine, pseudouridine, and $\mathrm{m}^{6} \mathrm{~A}$ in mRNAs.

Finally, it also seems likely that there will be new and startling discoveries about tRNA biology that are totally unanticipated. One need only look to the discoveries of RNA splicing, RNAi, and CRISPR, or to the discovery of a new code for translation of pseudouridine-containing mRNAs, to appreciate that many of the most exciting discoveries in RNA biology were completely unanticipated. It also seems highly likely based on past precedent that many new discoveries lie in wait from investigation of new organisms, new growth conditions, and different environmental nichesconditions where biology, evolution, and regulation take unusual twists - much like the unusual mitochondrial biology and the unusual biochemical activities that paved the way for the discovery of RNA editing.

\section{Acknowledgments}

We are grateful to Elizabeth Grayhack for helpful comments on the manuscript. This work was supported by NIH grants GM52347 to E.M.P. and GM27930 to A.K.H. 

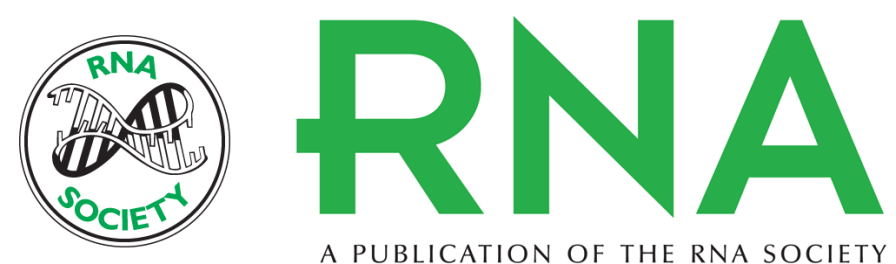

A PUBLICATION OF THE RNA SOCIETY

\section{tRNA processing, modification, and subcellular dynamics: past, present, and future}

Eric M. Phizicky and Anita K. Hopper

RNA 2015 21: 483-485

Open Access Freely available online through the RNA Open Access option.

Creative This article, published in $R N A$, is available under a Creative Commons License

Commons (Attribution-NonCommercial 4.0 International), as described at

License http://creativecommons.org/licenses/by-nc/4.0/.

Email Alerting Receive free email alerts when new articles cite this article - sign up in the box at the Service top right corner of the article or click here. 